\title{
Association of lesion size and visual prognosis to polypoidal choroidal vasculopathy.
}

\section{AUTHOR(S):}

Tsujikawa, Akitaka; Ojima, Yumiko; Yamashiro, Kenji; Nakata, Isao; Ooto, Sotaro; Tamura, Hiroshi; Nakanishi, Hideo; Hayashi, Hisako; Otani, Atsushi; Yoshimura, Nagahisa

\section{CITATION:}

Tsujikawa, Akitaka ...[et al]. Association of lesion size and visual prognosis to polypoidal choroidal vasculopathy.. American journal of ophthalmology 2011, 151(6): 961-972.e1

\section{ISSUE DATE:}

2011-06

URL:

http://hdl.handle.net/2433/142514

\section{RIGHT:}

@ 2011 Elsevier Inc.; この論文は出版社版でありません。引用の際には 出版社版をご確認ご利用ください。; This is not the published version. Please cite only the published version. 


\section{Abstract}

PURPOSE: To investigate the progression of vascular lesions of polypoidal choroidal vasculopathy (PCV) as viewed with indocyanine green angiography, and visual prognosis of these eyes.

DESIGN: Retrospective case study.

METHODS: We reviewed retrospectively the medical records of 88 consecutive patients (88 eyes) with PCV that had been examined with indocyanine green angiography for more than 2 years.

10 RESULTS: Depending on the initial area of the vascular lesion, eyes were divided into smaller PCV (baseline area of lesion being $<1$ disc area [DA], $n=22$ ) and larger PCV (baseline area of lesion being $\geq 1 \mathrm{DA}, \mathrm{n}=66$ ). In larger PCV, the mean area of the lesion progressed significantly from $6.49 \pm 8.96 \mathrm{~mm}^{2}$ to $16.27 \pm 14.19 \mathrm{~mm}^{2}(P<.0001)$ with marked deterioration of visual acuity $(P<.0001)$ during follow-up. In contrast, smaller

15 PCV often showed minimal progression of the lesion, only limited exudative change, and the eyes maintained their initially good vision to the final visit. Smaller PCV lesions rarely progressed to extensive PCV lesions. Severe vision threatening complications (i.e., suprachoroidal hemorrhage, vitreous hemorrhage, and tears of the retinal pigment epithelium) were seen only in eyes with larger PCV, and in studying SNPs A69S of ARMS2 genes, there was a significant difference in T allele frequency between individuals with smaller PCV and those with larger PCV $(20.2 \%$ vs $79.8 \%, P=.0235)$. CONCLUSIONS: PCV with small vascular lesions shows minimal progression and no vision threatening complications, and these eyes often maintain good visual acuity for a long time. 


\section{Association of Lesion Size and Visual Prognosis to Polypoidal Choroidal Vasculopathy}

AKITAKA TSUJIKAWA, MD, YUMIKO OJIMA, MD, KENJI YAMASHIRO, MD, ISAO NAKATA MD, SOTARO OOTO, MD, HIROSHI TAMURA, MD, HIDEO NAKANISHI, MD, HISAKO HAYASHI, MD, ATSUSHI OTANI, MD, AND NAGAHISA YOSHIMURA, MD

From the Department of Ophthalmology and Visual Sciences, Kyoto University Graduate School of Medicine, Kyoto, Japan.

Correspondence to Akitaka Tsujikawa, MD, Department of Ophthalmology, Kyoto University Graduate School of Medicine, Sakyo-ku, Kyoto 606-8507, Japan; fax: 20 +81-75-752-0933; e-mail: tujikawa@kuhp.kyoto-u.ac.jp 
Polypoidal choroidal vasculopathy (PCV) was first described as a new clinical entity with a unique form of choroidal vascular abnormality, ${ }^{1-3}$ and is characterized by a branching vascular network that terminates in polypoidal lesions seen by indocyanine green angiography. ${ }^{4}$ Initially, vascular components of PCV are reported to be seen

5 predominantly in a peripapillary location, ${ }^{5}$ but macular $\mathrm{PCV}^{6,7}$ and peripheral $\mathrm{PCV}^{5,8}$ have since been reported. Yannuzzi and associates ${ }^{9}$ expanded the clinical spectrum of this disease and established the current understanding of PCV. Today, macular PCV is more common in Asian populations and seems to be the condition most clinically significant. ${ }^{7,10,11}$ To date, however, the pathogenesis of PCV is not fully understood, and

10 it is still controversial as to whether or not it originates from an abnormality of the inner choroidal vessels or if it is a variant of choroidal neovascularization (CNV). ${ }^{12}$

PCV is accompanied often by recurrent serosanguineous detachments of the retinal pigment epithelium and neurosensory retina, and sometimes results in massive hemorrhagic complications with a sudden loss of vision. ${ }^{13,14}$ While the extent of visual

15 disturbance in PCV varies, it is generally thought that the visual prognosis of PCV is better than that of exudative age-related macular degeneration (AMD). ${ }^{11,12}$ In a previous report by Uyama and associates, ${ }^{11}$ about half of the patients with PCV had a favorable visual outcome (better than 20/30) after being followed-up for more than 2 years. In PCV, other vision-threatening complications, such as type $2 \mathrm{CNV}$, disciform scar, or cystoid 20 macular edema, are reported to be uncommon. ${ }^{10,12}$

Clinically, the size of the vascular lesions in PCV varies. ${ }^{15}$ We sometimes see cases of PCV with a large lesion that show a poor response to the treatment and show progression of the lesion, resulting in poor visual prognosis. Tateiwa and associates ${ }^{16}$ reported that PCV with a large vascular network that extends beyond the vascular 25 arcade is not uncommon, so we might speculate that vascular lesions of originally small PCV extend over time and result in these large PCV. Clinically, however, we rarely see 
this type of progression. ${ }^{17} \mathrm{PCV}$ cases with a small lesion often show minimal exudative change and no progression of the lesion, and can maintain good visual function for a long time. ${ }^{18}$ Even with an exudative change, small PCV often show a favorable response to treatment. ${ }^{19}$ Okubo and associates ${ }^{18}$ reported that a reddish-orange nodule alone, or

5 that multiple reddish-orange nodules with a small subretinal hemorrhage, is a sign of a potentially benign clinical course, so the clinical course of small and large PCV may be different.

In order to study the progression of vascular lesions in PCV, it is essential to perform repeated indocyanine green angiography, ${ }^{4}$ because most vascular lesions of

$10 \mathrm{PCV}$ are located beneath the retinal pigment epithelium. ${ }^{1-3}$ So far, however, there is little information on the long-term observation of the vascular components of PCV. ${ }^{15}$ In the study described herein, we investigated progression of the vascular lesion of PCV using indocyanine green angiography and visual prognosis of affected eyes. Based on our findings, we report a new classification of PCV and the expected complications and

15 visual prognosis of these two types of PCV.

\section{PATIENTS AND METHODS}

For this observational case study, we reviewed retrospectively the medical records of 88 consecutive patients (88 eyes) with symptomatic PCV who initially visited the Macula

20 Service of the Department of Ophthalmology at Kyoto University Hospital between January 2004 and October 2007, and who had been examined with both fluorescein and indocyanine green angiography for more than 2 years after their initial visit. When both eyes were diagnosed as having PCV, one eye was selected randomly for inclusion in the current study.

The diagnosis of PCV was based on indocyanine green angiography, which shows a branching vascular network that terminates in polypoidal swelling. The polypoidal 
lesion can be a single polyp or a cluster of multiple polyps. In most cases, the reddish-orange nodule that had been seen by the ophthalmoscopic examination corresponded to the polypoidal lesion. Eyes with other macular abnormalities (i.e., AMD, pathologic myopia, idiopathic CNV, presumed ocular histoplasmosis, angioid streaks, and other secondary CNV) were excluded from the current study. Eyes that were treated previously with focal laser photocoagulation, photodynamic therapy (PDT), vitrectomy, radiation therapy, or anti-vascular endothelial growth factor (VEGF) therapy were also excluded from the present study.

At the initial visit, all patients underwent a comprehensive ophthalmologic examination, including measurement of best-corrected visual acuity (VA), determination of intraocular pressure, indirect ophthalmoscopy, slitlamp biomicroscopy with a contact lens, and optical coherence tomography (OCT). After fundus photographs were taken, fluorescein and indocyanine green angiography were performed on each patient using a confocal laser scanning system (HRA-2, Heidelberg Engineering, Dossenheim,

15 Germany). In all patients, VA measurement and OCT examination were performed at each follow-up visit. At follow-up visits, angiography was performed if necessary, although all patients in the current study were examined with angiography several times during their follow-up. In the study described herein, the angiograms obtained at the initial visit were compared with the final angiograms.

In the current study, greatest linear dimension and area of the lesion were determined based on the indocyanine green angiography, using the software built into the HRA-2 machine. Greatest linear dimension included the entire PCV vascular lesion, including polypoidal lesion, branching vascular network vessels, and any type 2 CNV. The area of the vascular lesion was measured manually with the software that came with the HRA-2. The pigment epithelial detachment, without underlying vascular components, was not included in measurement of the greatest linear dimension and area of the lesion. 
In the current study, one optic-disc area (DA) is equal to $2.54 \mathrm{~mm}^{2}$, on the basis of one optic disc diameter being equal to $1.8 \mathrm{~mm}$. Based on the area of the lesion at the initial visit, we classified the eyes into either the smaller PCV group (baseline area of lesion $<1$ $\mathrm{DA}$ ) or the larger PCV group (baseline area of lesion $\geq 1 \mathrm{DA}$ ) in order to compare the clinical course of the two groups.

We also compared the initial OCT measurement and VA with values obtained at the final visit. To compare the difference in VA, VA measured with a Landolt chart was converted to a logarithm of the minimal angle of resolution (logMAR). Using OCT images, we performed two measurements (foveal thickness, and thickness of the neurosensory

10 retina in the fovea) with a caliper that was built into the software of the OCT machine. Foveal thickness was defined as the distance between the vitreoretinal interface and the retinal pigment epithelium; thickness of the neurosensory retina was defined as the distance between the vitreoretinal interface and the tip the outer segment of the inner and outer segments of the photoreceptors.

We genotyped the major AMD and PCV-associated SNP, rs10490924 (A69S), of ARMS2. Genomic DNA was prepared from leukocytes of peripheral blood using a DNA extraction kit (QuickGene-610L, Fujifilm, Minato, Tokyo, Japan). The SNPs were genotyped using Taqman SNP assays with the ABI PRISM 7700 system (Applied Biosystems, Foster City, California) according to the manufacturer's instructions.

Statistical analysis was performed using software designed for this purpose (StatView, version 5.0; SAS Institute, Cary, North Carolina). A P value of less than 0.05 was considered to be statistically significant.

\section{RESULTS}

25 In the current study, 88 eyes of 88 patients (60 men and 28 women) with PCV, ranging in age from 50 to 86 years $(70.4 \pm 7.5$ years), were examined. The follow-up period ranged 
from 29 to 61 months ( $46.4 \pm 8.6$ months), and duration from the initial angiogram to the last ranged from 24 to 60 months $(39.3 \pm 9.4$ months). All patients were examined with fluorescein and indocyanine green angiography repeatedly during follow-up, ranging from 2 to 11 times ( $4.9 \pm 2.0$ times). Table 1 shows the characteristics of patients eligible for inclusion in this study. The mean baseline VA (logMAR) was $0.37 \pm 0.34$. The mean initial area of the lesion and greatest linear dimension was $7.75 \pm 9.78 \mathrm{~mm}^{2}$ and $3412 \pm$ $1647 \mu \mathrm{m}$, respectively. Figure 1 shows the relationship between area of the lesion, greatest linear dimension, foveal thickness, and VA at initial visit and final examination. Initial area of the lesion $(\mathrm{R}=0.801, P<.0001)$ and initial greatest linear dimension $(\mathrm{R}=$ 0.805, $P<.0001$ ) showed a close correlation with final measurements.

PCV vascular lesion at the initial visit varied in size, ranging from 0.64 to 63.82 $\mathrm{mm}^{2}$. Depending on the initial area of the lesion, we divided the eyes with PCV into two groups-the smaller PCV group (baseline area of lesion being $<1$ DA, $n=22$ ) and the larger PCV group (baseline area of lesion being $\geq 1 \mathrm{DA}, \mathrm{n}=66$ ) (Figure 2). The mean

15 area of the lesion was initially $1.68 \pm 0.53 \mathrm{~mm}^{2}$ in the smaller PCV group and $9.79 \pm$ $10.55 \mathrm{~mm}^{2}$ in the larger PCV group. There were no significant differences in gender, age, or duration of symptoms between groups $(P=.5971, P=.3257$, and $P=.6484$, respectively). In addition, there were no differences in the foveal thickness $(P=.4628)$ or thickness of the neurosensory retina in the fovea $(P=.4204)$ at the initial visit. However,

20 the initial VA was significantly better in eyes with smaller PCV $(0.24 \pm 0.39)$ than in eyes with larger PCV $(0.42 \pm 0.35, P=.0383)$.

During the follow-up period, 64 eyes were treated initially with PDT, and 9 were treated initially with anti-VEGF therapy. In spite of these treatments, some eyes with larger PCV showed extension of the vascular component with an exudative change. The 25 mean area of the lesion in larger PCV progressed significantly from $9.79 \pm 10.55 \mathrm{~mm}^{2}$ to $16.27 \pm 14.19 \mathrm{~mm}^{2}$ at the final examination $(P<.0001)$ (Figure 3). Furthermore, VA in 
these eyes deteriorated significantly $0.42 \pm 0.35$ to $0.76 \pm 0.49$ at the final examination $(P<.0001)$. In contrast, eyes with smaller PCV lesions often showed minimal progression of the lesion and limited exudative change, and smaller PCV lesions rarely progressed to extensive PCV lesions (Figure 4). However, even in eyes with smaller PCV, the mean lesion size increased during the follow-up period $(P=.0037)$. In smaller PCV, change in area of the lesion and final area of the lesion were $2.45 \pm 3.53 \mathrm{~mm}^{2}$ and $4.13 \pm 3.59 \mathrm{~mm}^{2}$, respectively, which were significantly less than those of the larger PCV $(P=.0429$ and $P=.0002$, respectively). In addition, eyes with the smaller PCV showed no decrease in VA $(-0.05 \pm 0.36, P=.5492)$, and maintained initial VA to the final visit;

10 mean changes in VA were significantly better in smaller PCV than were those in larger $\operatorname{PCV}(P=.0015)$.

Table 2 shows the ocular manifestations and complications seen during follow-up in eyes with smaller or larger PCV. Of the 88 eyes included, seven (7.6\%) showed suprachoroidal hemorrhage and eight (8.7\%) showed vitreous hemorrhage (Figure 5),

15 all of which were seen in eyes with larger PCV; no eyes with smaller PCV showed severe complications $(P=.1111$ and $P=.0868)$. Indeed, in eyes with smaller $P C V$, even the relatively small amount of subretinal hemorrhage noted (> 1 DA) was seen less frequently than in eyes with larger PCV $(P=.0157)$. In addition, other ocular manifestations associated with severe visual disturbance were seen more often in eyes with larger PCV. Type 2 CNV, subretinal fibrosis, and cystoid macular edema were seen more frequently in eyes with larger $\operatorname{PCV}(P=.0030, P=.0533$, and $P=.0266)$. Of the 88 eyes included in this study, 41 (46.6\%) showed a serosanguineous pigment epithelial detachment (area > 1 DA). Again, a pigment epithelial detachment was seen more frequently in eyes with larger PCV than in those with smaller PCV $(P=.0096)$. Of the 88

25 eyes of our patients, eight (8.7\%) showed a tear of the retinal pigment epithelium. All of these occurred in eyes with larger PCV; no eyes with smaller PCV showed a tear ( $P$ 
$=.0868)$.

We were able to examine the DNA of 76 of the 88 patients. Table 3 shows the distributions of ARMS2 (A69S) genotypes in patients with smaller PCV and in those with larger PCV. There was a significant difference in T allele frequency between patients with smaller PCV and those with larger PCV $(20.2 \%$ vs $79.8 \%, P=.0235)$. In comparison to wild-type homozygosity (GG), homozygosity for the at-risk genotype (TT) increased the likelihood for the larger PCV group by 5.0-fold, and heterozygosity for the at-risk genotype (GT) increased the likelihood for this same group by 1.5 -fold.

\section{DISCUSSION}

Based on the initial area of the vascular lesion, we defined smaller PCV as those with a baseline area of $<1 \mathrm{DA}$. The remaining PCV, in which the baseline area was $\geq 1 \mathrm{DA}$, were defined as larger PCV. There were no significant differences in age or duration of symptoms between these two groups. The larger PCV did, however, often show

15 progression of the vascular lesions, which in many instances showed an exudative change. In larger PCV, poor initial VA was even further lessened in spite of the treatment. In contrast, most eyes diagnosed as having smaller PCV showed only minimal progression of the lesion, and limited exudative change. Furthermore, smaller PCV lesions rarely progressed to extensive PCV lesions. Most eyes with smaller PCV had

20 good initial VA, and maintained their good VA throughout the follow-up period.

In addition, severe complications such as suprachoroidal hemorrhage, vitreous hemorrhage, and a tear of the retinal pigment epithelium were seen only in eyes with larger PCV; no eyes with smaller PCV showed these severe complications. Furthermore, type 2 CNV and subfoveal fibrosis was seen more frequently in larger PCV. From these

25 findings, we believe that the ocular manifestations, complications, and visual prognosis of smaller PCV are distinct from those of larger PCV. Okubo and associates ${ }^{18}$ reported 
similar findings. Of 13 eyes with PCV that were followed-up for 5 years or longer, with no treatment, they reported that eyes with reddish-orange nodules alone or those with nodules plus a small subretinal hemorrhage had a benign clinical course with stable vision. $^{18}$

PCV is thought to have a better visual prognosis than does exudative AMD, ${ }^{9,20}$ although the visual prognosis in PCV is not as promising as was thought initially. ${ }^{21}$ Previously, direct laser photocoagulation was applied to eyes with PCV. ${ }^{7,22}$ Unless the entire vascular lesions could be coagulated, however, the polypoidal lesion often recurred, resulting in decreased VA, ${ }^{23}$ although another study showed encouraging

10 short-term results of PDT for PCV. ${ }^{24}$ However, one or more years after initially successful treatment with PDT, some eyes had a recurrence of PCV and a decrease in VA. ${ }^{21}$ Still more recently, although anti-VEGF therapy can reduce the exudative change in PCV shortly after treatment, its effects on the vascular lesions and its effect on VA appear to be limited. ${ }^{25-29}$ In addition, some eyes with large vascular lesions show massive

15 hemorrhagic complications, with sudden visual loss. ${ }^{30}$ As shown in Table 2, other vision-threatening complications, such as disciform scar, a tear of the retinal pigment epithelium, or cystoid macular edema, are not as uncommon as reported initially. ${ }^{16,31,32}$ Smaller PCV, which often shows a favorable response to treatments and with minimal vision-threatening complications, turn out to have a better visual prognosis than do 20 larger PCV. ${ }^{17,18}$

So far, several classifications of PCV have been reported. ${ }^{11,12}$ Based on the location of the lesion, PCV can be categorized as peripapillary, macular, or peripheral. ${ }^{12}$ When the vascular lesion is located far from the fovea, visual prognosis tends to be good. ${ }^{5}$ Uyama and associates ${ }^{11}$ reported two patterns of fundus manifestation of PCV,

25 exudative and hemorrhagic. However, they reported also that $36 \%$ of the cases had altered their pattern of manifestation during follow-up. ${ }^{11}$ Judging from these patterns, it 
might be difficult to predict visual prognosis.

The polypoidal lesions are thought to be the main source of the exudative change and hemorrhagic complications in PCV. ${ }^{4,14}$ Uyama and associates ${ }^{11}$ reported two patterns of polypoidal lesions: solitary round aneurysmal dilations and a collection of small aneurysmal dilations that resemble a cluster of grapes, the latter of which is associated with a poor prognosis. Clinically, some polypoidal lesions do regress spontaneously, and regress even more after PDT, but these polypoidal lesions recur at the same location or at other terminals of the branching vascular network. ${ }^{9,12,33}$ Recently, Cackett and associates ${ }^{34}$ reported a classification system of PCV based on polypoidal

10 lesion seen by indocyanine green angiography. In their report, ${ }^{34}$ PCV was classified according to size, location, formation, and number of polypoidal lesions. While objective evaluations are essential for any multicenter study, the branching vascular network, which is one origin of the polypoidal lesions, is essential also to understand status of the disease in each individual patient. ${ }^{35}$

Based of the observation by indocyanine green angiography, Yuzawa and associates $^{36}$ reported that there may be two types of branching vascular network, one representing an intrachoroidal vascular abnormality, and the other representing neovascularization that grows rapidly in the subretinal pigment epithelial space. Unfortunately, histologic reports of PCV are limited, ${ }^{37,38}$ although recent OCT findings support the theory that the vascular components of PCV are located intra- or supra-Bruch's membrane. In Figure 4, both the polypoidal lesion and the branching vascular network are seen between the retinal pigment epithelium and Bruch's membrane, ${ }^{39}$ suggesting that the vascular lesions in PCV are a form of CNV. ${ }^{14}$

The branching vascular network, which only rarely disappeared with treatment, 25 tended to extend over time. ${ }^{35}$ It is thought that progression of PCV is slower than that of exudative AMD. ${ }^{9}$ Yannuzzi and associates ${ }^{9}$ reported that the branching vascular network 
enlarges by simple proliferation and hypertrophy of the vascular components, by conversion of the polypoidal lesion into the advancing edge of a vascular channel, or by the unfolding of a cluster of polypoidal lesions and subsequent transformation into enlarging vascular tubular components. In the current study, based on the initial size of the vascular components, we divided PCV into smaller and larger types, a classification that provides useful information to both physicians and patients on the risk of severe complications and on visual prognosis. Smaller PCV rarely progress to become extensive lesions, and often have a more favorable clinical course that is quite different from that of larger PCV. ${ }^{17,18}$ In the current study, all 15 eyes that developed finally

10 extensive vascular lesions (more than $8 \mathrm{DA}$ ) had the lesion of more than $3 \mathrm{DA}$ at the initial examination. It follows that all eyes with extensive PCV had already shown relatively large vascular lesion when they had initial visual symptoms. From the current study, we could not provide any information on the beginning of the extensive PCV.

In the current study, we examined the genotypes of rs10490924 (A69S) of ARMS2

15 in patients with smaller PCV (21 patients) and those with larger PCV (55 patients). An increasing number of reports showed that ARMS2 A69S is associated strongly with AMD, as well as with typical AMD and with PCV. ${ }^{40-45}$ In the current study, there was a significant difference in T allele frequency between the smaller PCV and the larger PCV (20.2\% vs $79.8 \%$ ). Our findings suggest that smaller PCV is different from larger PCV, not only from the clinical point of view but also from the genetic point of view.

In PCV patients, Sakurada and associates ${ }^{40}$ reported a difference in the genotypic frequency at this site between eyes with and without vitreous hemorrhage, with the frequency of the $T$ allele being significantly greater in the vitreous hemorrhage group than that in the non-vitreous hemorrhage group. ${ }^{40}$ In another report, Sakurada and associates ${ }^{46}$ reported that this genotype is not associated with lesion composition or size as seen by indocyanine green angiography. However, they did indicate that the T allele 
at this SNP is associated with the exudative activity of polypoidal lesions. ${ }^{46}$ While further studies are necessary to elucidate the contribution of this SNP to the progression of the vascular lesion, this SNP appears to be associated with the occurrence of PCV itself and with activity of the vascular lesions.

Limitations of the current study are its retrospective nature and the various treatment regimens used. Our patents received primarily PDT or anti-VEGF therapy. ${ }^{25-29}$ Because recent studies have suggested that PDT in combination with anti-VEGF therapy may be the most promising treatment of $\mathrm{PCV}$, it is possible that our patients did not receive the most effective treatment. ${ }^{47,48}$ In the current study, 8 eyes showed a

10 relatively large subretinal hemorrhage at the initial visit. It may be possible that the lesion size was somewhat underestimated in a few eyes. However, because all of these eight eyes were classified as having larger PCV, there is no possibility that any eye with a relatively large subretinal hemorrhage was classified incorrectly as having a smaller PCV. In addition, 11 of 22 eyes with smaller PCV still had small lesions (<1 DA) at the

15 final examination. In remaining 11 eyes, however, final area of the lesion was greater than $1 \mathrm{DA}$, although the lesion remained relatively small and visual prognosis was often good. If these eyes had been examined later, they might be classified as having larger PCV. However, despite these shortcomings, our findings suggest that most smaller PCV show limited progression and that good visual function is maintained-with no serious

20 complications. Furthermore, it may be of use to differentiate this type of PCV from larger PCV in order to prognosticate visual prognosis in affected individuals.

\section{ACKNOWLEDGMENTS}

A. Funding/Support: This study was supported in part by the Japan Society for the

25 Promotion of Science (JSPS), Tokyo, Japan (Grant-in-Aid for Scientific Research, no. 21592256), and the Japan National Society for the Prevention of Blindness, Tokyo, 
Japan.

B. Financial Disclosure: None.

C. Contributions of Authors: Conception and design of the study (AT, YO, NY); analysis and interpretation ( $\mathrm{KY}, \mathrm{IN}, \mathrm{SO}, \mathrm{HT}, \mathrm{HN}, \mathrm{HH}, \mathrm{AO})$; writing of the article (AT, YO); critical revision of the article $(\mathrm{KY}, \mathrm{IN}, \mathrm{SO}, \mathrm{HT}, \mathrm{HN}, \mathrm{HH}, \mathrm{AO}, \mathrm{NY})$; final approval of the article (AT, YO, KY, IN, SO, HT, HN, HH, AO, NY); data collection (AT, YO, KY, IN, SO, $\mathrm{HT}, \mathrm{HN}, \mathrm{HH}, \mathrm{AO})$.

D. Ethics Committee Approval: This study was approved by the Institutional Review Board at Kyoto University Graduate School of Medicine and adhered to the tenets of the

10 Declaration of Helsinki. In the guidelines, it is not mandatory to obtain informed consent from patients for a retrospective study in which the researchers review only medical records.

E. Other Acknowledgments: None.

\section{REFERENCES}

1. Kleiner RC, Brucker AJ, Johnston RL. The posterior uveal bleeding syndrome. Retina 1990;10(1):9-17.

2. Stern RM, Zakov ZN, Zegarra H, Gutman FA. Multiple recurrent serosanguineous retinal pigment epithelial detachments in black women. Am J Ophthalmol 1985;100(4):560-569.

3. Yannuzzi LA, Sorenson J, Spaide RF, Lipson B. Idiopathic polypoidal choroidal vasculopathy (IPCV). Retina 1990;10(1):1-8.

4. Spaide RF, Yannuzzi LA, Slakter JS, Sorenson J, Orlach DA. Indocyanine green videoangiography of idiopathic polypoidal choroidal vasculopathy. Retina 1995;15(2):100-110.

5. Yannuzzi LA, Nogueira FB, Spaide RF, et al. Idiopathic polypoidal choroidal 
vasculopathy: a peripheral lesion. Arch Ophthalmol 1998;116(3):382-383.

6. Moorthy RS, Lyon AT, Rabb MF, Spaide RF, Yannuzzi LA, Jampol LM. Idiopathic polypoidal choroidal vasculopathy of the macula. Ophthalmology 1998;105(8):1380-1385.

7. Uyama M, Matsubara T, Fukushima I, et al. Idiopathic polypoidal choroidal vasculopathy in Japanese patients. Arch Ophthalmol 1999;117(8):1035-1042.

8. Akman A, Akbatur H, Yilmaz G, Aydin P. Peripheral idiopathic polypoidal choroidal vasculopathy. Eye 2000;14(Pt 4):680-681.

9. Yannuzzi LA, Ciardella A, Spaide RF, Rabb M, Freund KB, Orlock DA. The expanding clinical spectrum of idiopathic polypoidal choroidal vasculopathy. Arch Ophthalmol 1997;115(4):478-485.

10. Sho K, Takahashi K, Yamada H, et al. Polypoidal choroidal vasculopathy: incidence, demographic features, and clinical characteristics. Arch Ophthalmol 2003;121(10):1392-1396.

15 11. Uyama M, Wada M, Nagai Y, et al. Polypoidal choroidal vasculopathy: natural history. Am J Ophthalmol 2002;133(5):639-648.

12. Ciardella AP, Donsoff IM, Huang SJ, Costa DL, Yannuzzi LA. Polypoidal choroidal vasculopathy. Surv Ophthalmol 2004;49(1):25-37.

13. Ahuja RM, Stanga PE, Vingerling JR, Reck AC, Bird AC. Polypoidal choroidal vasculopathy in exudative and haemorrhagic pigment epithelial detachments. $\mathrm{Br} \mathrm{J}$ Ophthalmol 2000;84(5):479-484.

14. Tsujikawa A, Sasahara M, Otani A, et al. Pigment epithelial detachment in polypoidal choroidal vasculopathy. Am J Ophthalmol 2007;143(1):102-111.

15. Gomi F, Sawa M, Mitarai K, Tsujikawa M, Tano Y. Angiographic lesion of polypoidal choroidal vasculopathy on indocyanine green and fluorescein angiography. Graefes Arch Clin Exp Ophthalmol 2007;245(10):1421-1427. 
16. Tateiwa H, Kuroiwa S, Gaun S, Arai J, Yoshimura N. Polypoidal choroidal vasculopathy with large vascular network. Graefes Arch Clin Exp Ophthalmol 2002;240(5):354-361.

17. Okubo A, Hirakawa M, Ito M, Sameshima M, Sakamoto T. Clinical features of early and late stage polypoidal choroidal vasculopathy characterized by lesion size and disease duration. Graefes Arch Clin Exp Ophthalmol 2008;246(4):491-499.

18. Okubo A, Arimura N, Abematsu N, Sakamoto T. Predictable signs of benign course of polypoidal choroidal vasculopathy: based upon the long-term observation of non-treated eyes. Acta Ophthalmol 2010;88(4):107-114.

19. Okubo A, Ito M, Kamisasanuki T, Sakamoto T. Visual improvement following trans-Tenon's retrobulbar triamcinolone acetonide infusion for polypoidal choroidal vasculopathy. Graefes Arch Clin Exp Ophthalmol 2005;243(8):837-839.

20. Maruko I, lida T, Saito M, Nagayama D, Saito K. Clinical characteristics of exudative age-related macular degeneration in Japanese patients. Am J Ophthalmol 2007;144(1):15-22.

21. Kurashige $Y$, Otani A, Sasahara M, et al. Two-year results of photodynamic therapy for polypoidal choroidal vasculopathy. Am J Ophthalmol 2008;146(4):513-519.

22. Nishijima K, Takahashi M, Akita J, et al. Laser photocoagulation of indocyanine green angiographically identified feeder vessels to idiopathic polypoidal choroidal vasculopathy. Am J Ophthalmol 2004;137(4):770-773.

23. Yuzawa M, Mori R, Haruyama M. A study of laser photocoagulation for polypoidal choroidal vasculopathy. Jpn J Ophthalmol 2003;47(4):379-384.

24. Chan WM, Lam DS, Lai TY, et al. Photodynamic therapy with verteporfin for symptomatic polypoidal choroidal vasculopathy: one-year results of a prospective case series. Ophthalmology 2004;111(8):1576-1584.

25. Lee SY, Kim JG, Joe SG, Chung H, Yoon YH. The therapeutic effects of 
bevacizumab in patients with polypoidal choroidal vasculopathy. Korean $\mathrm{J}$ Ophthalmol 2008;22(2):92-99.

26. Gomi F, Sawa M, Sakaguchi H, et al. Efficacy of intravitreal bevacizumab for polypoidal choroidal vasculopathy. Br J Ophthalmol 2008;92(1):70-73.

27. Lai TY, Chan WM, Liu DT, Luk FO, Lam DS. Intravitreal bevacizumab (Avastin) with or without photodynamic therapy for the treatment of polypoidal choroidal vasculopathy. Br J Ophthalmol 2008;92(5):661-666.

28. Tsujikawa A, Ooto S, Yamashiro K, Tamura H, Otani A, Yoshimura N. Treatment of polypoidal choroidal vasculopathy by intravitreal injection of bevacizumab. Jpn J Ophthalmol 2010;54(4):310-319.

29. Kokame GT, Yeung L, Lai JC. Continuous anti-VEGF treatment with ranibizumab for polypoidal choroidal vasculopathy: 6-month results. $\mathrm{Br} \mathrm{J}$ Ophthalmol 2010;94(3):297-301 .

30. Hirami Y, Tsujikawa A, Otani A, et al. Hemorrhagic complications after photodynamic therapy for polypoidal choroidal vasculopathy. Retina 2007;27(3):335-341.

31. Tsujikawa A, Hirami Y, Nakanishi $\mathrm{H}$, et al. Retinal pigment epithelial tear in polypoidal choroidal vasculopathy. Retina 2007;27(7):832-838.

32. Tamura H, Tsujikawa A, Otani A, et al. Polypoidal choroidal vasculopathy appearing as classic choroidal neovascularisation on fluorescein angiography. $\mathrm{Br} \mathrm{J}$ Ophthalmol 2007;91(9):1152-1159.

33. Otani A, Sasahara M, Yodoi Y, et al. Indocyanine green angiography: guided photodynamic therapy for polypoidal choroidal vasculopathy. Am J Ophthalmol 2007;144(1):7-14.

34. Cackett P, Wong D, Yeo I. A classification system for polypoidal choroidal vasculopathy. Retina 2009;29(2):187-191. 
35. Lim TH, Laude A, Tan CS. Polypoidal choroidal vasculopathy: an angiographic discussion. Eye 2010;24(3):483-490.

36. Yuzawa M, Mori R, Kawamura A. The origins of polypoidal choroidal vasculopathy. Br J Ophthalmol 2005;89(5):602-607.

5 37. Lafaut BA. Clinicopathologic correlation of surgically removed submacular tissue. Bull Soc Belge Ophtalmol 2000(278):49-53.

38. Rosa RH Jr, Davis JL, Eifrig CW. Clinicopathologic reports, case reports, and small case series: clinicopathologic correlation of idiopathic polypoidal choroidal vasculopathy. Arch Ophthalmol 2002;120(4):502-508.

10 39. Ojima Y, Hangai M, Sakamoto A, et al. Improved visualization of polypoidal choroidal vasculopathy lesions using spectral-domain optical coherence tomography. Retina 2009;29(1):52-59.

40. Sakurada Y, Kubota T, Mabuchi F, Imasawa M, Tanabe N, lijima H. Association of LOC387715 A69S with vitreous hemorrhage in polypoidal choroidal vasculopathy. Am J Ophthalmol 2008;145(6):1058-1062.

41. Lima LH, Schubert C, Ferrara DC, et al. Three major loci involved in age-related macular degeneration are also associated with polypoidal choroidal vasculopathy. Ophthalmology 2010;117(8):1567-1570.

42. Mori K, Horie-Inoue K, Gehlbach PL, et al. Phenotype and genotype characteristics of age-related macular degeneration in a Japanese population. Ophthalmology 2010;117(5):928-938.

43. Lee KY, Vithana EN, Mathur R, et al. Association analysis of $\mathrm{CFH}, \mathrm{C2}$, BF, and HTRA1 gene polymorphisms in Chinese patients with polypoidal choroidal vasculopathy. Invest Ophthalmol Vis Sci 2008;49(6):2613-2619. in polypoidal choroidal vasculopathy and age-related macular degeneration in a 
Japanese population. Am J Ophthalmol 2007;144(4):608-612.

45. Gotoh N, Nakanishi H, Hayashi H, et al. ARMS2 (LOC387715) variants in Japanese patients with exudative age-related macular degeneration and polypoidal choroidal vasculopathy. Am J Ophthalmol 2009;147(6):1037-1041.

46. Sakurada $Y$, Kubota $T$, Imasawa M, et al. Angiographic lesion size associated with LOC387715 A69S genotype in subfoveal polypoidal choroidal vasculopathy. Retina 2009;29(10):1522-1526.

47. Ogino T, Takeda M, Imaizumi H, Okushiba U. Photodynamic therapy for age-related macular degeneration in Japanese patients: results after one year. Jpn J Ophthalmol 2007;51(3):210-215.

48. Sato T, Kishi S, Matsumoto H, Mukai R. Combined photodynamic therapy with verteporfin and intravitreal bevacizumab for polypoidal choroidal vasculopathy. Am J Ophthalmol 2010;149(6):947-954.

Figure 1. Scattergrams showing area of the lesion, greatest linear dimension, foveal thickness, and visual acuity (VA) in eyes with polypoidal choroidal vasculopathy (PCV) obtained at initial and final examinations. Initial area (upper left) is correlated significantly with final area of the lesion $(R=0.801, P<.0001)$. Initial greatest linear dimension

20 (upper right) is correlated significantly with final greatest linear dimension $(R=0.805, P$ $<.0001)$. Correlations between initial and final foveal thickness (bottom left) $(R=0.316$, $P=.0025)$, and initial and final VA (bottom right) $(R=0.355, P=.0006)$. VA measured with a Landolt chart was converted to a logarithm of the minimal angle of resolution $(\log M A R)$.

Figure 2. Indocyanine green angiograms obtained at the initial visit from eyes with 
polypoidal choroidal vasculopathy (PCV). All eyes showed the branching vascular network that terminated in a polypoidal lesion, although the lesions varied in size. (Upper) Indocyanine green angiograms in the group with smaller PCV. (Bottom) Indocyanine green angiograms in the group with larger PCV.

Figure 3. Progression of the vascular lesion in larger polypoidal choroidal vasculopathy (PCV). (Upper left) Fundus photograph at the initial visit shows a reddish orange nodule with a minute pigment epithelial detachment (PED). (Upper right) A sectional image obtained with optical coherence tomography (OCT) along with an arrow seen in the

10 fundus photograph shows a small protrusion of the retinal pigment epithelium corresponding to the PED. (2nd row, left) Fluorescein angiography at the initial visit shows occult choroidal neovascularization corresponding to a branching vascular network. (2nd row, right) Indocyanine green angiography reveals large vascular components of PCV, consisting of a polypoidal lesion (arrow) and a branching vascular

15 network (long arrow). The area of the PCV lesion was $5.87 \mathrm{~mm}^{2}$. (3rd row, left) In spite of three anti-VEGF treatments, the vascular lesion progressed. Fundus photograph obtained at 33 months after the initial visit shows a large serosanguineous PED. (3rd row, right) A sectional image obtained by OCT (with the arrow shown in the fundus photograph) shows a steep protrusion of retinal pigment epithelium, which is reflective of

20 the large PED. (Bottom left) Fluorescein angiography shows occult choroidal neovascularization corresponding to the branching vascular network. (Bottom right) indocyanine green angiography shows progression of the polypoidal lesions and extension of the branching vascular network. The area of the PCV lesion increased to $9.40 \mathrm{~mm}^{2}$.

Figure 4. No progression of the vascular lesion in smaller polypoidal choroidal 
vasculopathy (PCV). (Upper left) Fundus photograph at the initial visit shows a small reddish orange nodule (arrow); vision was 20/16. (Upper middle) Fluorescein angiography (FA) at the initial visit shows only a hyperfluorescent spot corresponding to the polypoidal lesion. (Upper right) Indocyanine green angiography shows the vascular components of PCV, which consist of a typical polypoidal lesion (arrow) and a branching vascular network (long arrow). The baseline area of the PCV lesion was $1.77 \mathrm{~mm}^{2}$. (2nd row) A sectional image obtained with optical coherence tomography (OCT) along with the arrow seen in the FA image shows a steep protrusion of the retinal pigment epithelium. (3rd row, left) No treatment was performed. Fundus photograph obtained at

1039 months after the initial visit shows a reddish orange nodule with a newly developed serous pigment epithelial detachment; vision was still 20/13. (3rd row, middle) FA shows a hyperfluorescent spot that corresponds to the polypoidal lesion, along with fluorescein pooling in the pigment epithelial detachment. (3rd row, right) Indocyanine green angiography reveals no progression of the vascular lesion of PCV. (Bottom) A sectional

15 image obtained with OCT (with the arrow seen on FA) shows protrusion of the retinal pigment epithelium that corresponds to the branching network (arrowheads) and steep elevation of the retinal pigment epithelium with moderate inner reflectivity (arrow) corresponding to the polypoidal lesion.

20 Figure 5. Suprachoroidal hemorrhage and vitreous hemorrhage in an eye with larger polypoidal choroidal vasculopathy (PCV). (Upper) Fundus photograph at the initial visit shows multiple large reddish orange nodules; vision was 20/22. Indocyanine green angiography shows a large branching vascular network that terminates with multiple polypoidal lesions. The baseline area of the PCV lesion was $15.80 \mathrm{~mm}^{2}$. (Middle) No treatment was performed because this eye had good visual acuity. Fundus photograph obtained at 29 months after the initial visit shows some extension of the vascular 
components; vision was 20/16. Indocyanine green angiography shows some extension of the vascular lesion of PCV. (Bottom) Five months after the last angiogram, the eye developed a sudden suprachoroidal hemorrhage with subsequent vitreous hemorrhage. After vitreous surgery, visual acuity in this eye was only 20/200. 
TABLE 1. Characteristics of Patients with Polypoidal Choroidal Vasculopathy

\begin{tabular}{|c|c|c|c|c|}
\hline & $\begin{array}{c}\text { Total } \\
(\mathrm{n}=88)\end{array}$ & $\begin{array}{c}\text { Smaller } \\
\text { Polypoidal } \\
\text { Choroidal } \\
\text { Vasculopathy } \\
(n=22)\end{array}$ & $\begin{array}{c}\text { Larger } \\
\text { Polypoidal } \\
\text { Choroidal } \\
\text { Vasculopathy } \\
(n=66)\end{array}$ & $P$ value \\
\hline Gender (women/men) & $28 / 60$ & $8 / 14$ & $20 / 46$ & .5971 \\
\hline Age (years) & $70.4 \pm 7.5$ & $68.2 \pm 6.9$ & $70.8 \pm 7.7$ & .3257 \\
\hline Hypertension & 38 & 11 & 27 & .4560 \\
\hline Smoking & 14 & 4 & 10 & .7365 \\
\hline \multicolumn{5}{|l|}{ Location of lesions } \\
\hline (macular/peripapillary/peripheral) & $79 / 8 / 1$ & $22 / 0 / 0$ & $57 / 8 / 1$ & .1881 \\
\hline Duration of symptoms (months) & $8.0 \pm 11.9$ & $7.0 \pm 9.5$ & $8.3 \pm 12.6$ & .6484 \\
\hline \multicolumn{5}{|l|}{ Initial conditions } \\
\hline Best-corrected visual acuity (logMAR) & $0.37 \pm 0.34$ & $0.24 \pm 0.29$ & $0.42 \pm 0.35$ & .0383 \\
\hline Area of lesion $\left(\mathrm{mm}^{2}\right)$ & $7.75 \pm 9.78$ & $1.68 \pm 0.53$ & $9.79 \pm 10.55$ & .0006 \\
\hline Greatest linear dimension $(\mu \mathrm{m})$ & $3412 \pm 1647$ & $1901 \pm 464$ & $3915 \pm 1591$ & $<.0001$ \\
\hline Foveal thickness $(\mu \mathrm{m})$ & $403.5 \pm 189.9$ & $377.6 \pm 175.4$ & $412.2 \pm 195.0$ & .4628 \\
\hline $\begin{array}{l}\text { Thickness of neurosensory retina in the } \\
\text { fovea }(\mu \mathrm{m})\end{array}$ & $196.5 \pm 83.8$ & $209.1 \pm 98.5$ & $192.4 \pm 78.6$ & .4204 \\
\hline Follow-up period (months) & $46.4 \pm 8.1$ & $44.5 \pm 6.7$ & $47.0 \pm 8.4$ & .2020 \\
\hline \multicolumn{5}{|l|}{ Treatment } \\
\hline Photodynamic therapy & 69 & 16 & 53 & .3055 \\
\hline (Times of treatments) & $1.9 \pm 1.1$ & $1.5 \pm 0.7$ & $2.1 \pm 1.2$ & .0875 \\
\hline Anti-VEGF therapy & 40 & 6 & 34 & .0480 \\
\hline (Times of treatments) & $2.9 \pm 2.5$ & $3.8 \pm 3.7$ & $2.7 \pm 2.2$ & .3155 \\
\hline Pars plana vitrectomy & 4 & 0 & 4 & .2372 \\
\hline Cataract surgery & 8 & 3 & 5 & .2554 \\
\hline
\end{tabular}

Final conditions
Best-corrected visual acuity (logMAR)
Area of lesion $\left(\mathrm{mm}^{2}\right)$
Greatest linear dimension $(\mu \mathrm{m})$
Foveal thickness $(\mu \mathrm{m})$

Thickness of neurosensory retina in the fovea $(\mu \mathrm{m})$

$0.62 \pm 0.51$
$13.24 \pm 13.47$
$4511 \pm 2030$
$299.7 \pm 189.5$

$0.19 \pm 0.33$

$4.13 \pm 3.59$

$2761 \pm 900$

$235.3 \pm 65.1$

$197.1 \pm 168.1$

$153.3 \pm 38.4$

$211.7 \pm 191.1$

.1597

Changes during follow-up

$\begin{array}{lllll}\text { Best-corrected visual acuity }(\log M A R) & 0.24 \pm 0.51 & -0.05 \pm 0.36 & 0.34 \pm 0.51 & .0015 \\ \text { Area of lesion }\left(\mathrm{mm}^{2}\right) & 5.48 \pm 8.13 & 2.45 \pm 3.53 & 6.49 \pm 8.96 & .0429\end{array}$


Greatest linear dimension $(\mu \mathrm{m})$

$$
\begin{array}{cccc}
1100 \pm 1204 & 860 \pm 933 & 1180 \pm 1278 & .2838 \\
-103.8 \pm 221.9 & -142.3 \pm 163.6 & -91.0 \pm 237.8 & .3500
\end{array}
$$

Foveal thickness $(\mu \mathrm{m})$

Thickness of neurosensory retina in the fovea $(\mu \mathrm{m})$

$\mathrm{n}=$ number; $\log M A R=$ logarithm of the minimum angle of resolution; VEGF = vascular endothelial growth factor. One disc area (DA) is estimated as $2.54 \mathrm{~mm}^{2}$ on the basis of the one optic disc diameter of $1.8 \mathrm{~mm}$. Based on the area of lesion at the initial visit, polypoidal choroidal vasculopathy (PCV) eyes were divided into smaller PCV (area of lesion $<1 \mathrm{DA}$ ) and larger PCV (area of lesion $\geq 1 \mathrm{DA}$ ). 
TABLE 2. Ocular Manifestations and Complications Seen in Eyes with Polypoidal Choroidal Vasculopathy During Follow-up

\begin{tabular}{|c|c|c|c|c|}
\hline & \multirow{5}{*}{$\begin{array}{c}\text { Total } \\
(\mathrm{n}=88)\end{array}$} & \multirow{3}{*}{$\begin{array}{l}\text { Smaller } \\
\text { Polypoidal } \\
\text { Choroidal }\end{array}$} & \multicolumn{2}{|l|}{ Larger } \\
\hline & & & \multicolumn{2}{|l|}{ Polypoidal } \\
\hline & & & \multicolumn{2}{|l|}{ Choroidal } \\
\hline & & Vasculopathy & \multicolumn{2}{|l|}{ Vasculopathy } \\
\hline & & $(n=22)$ & $(n=66)$ & $P$ value \\
\hline Suprachoroidal hemorrhage & 7 & 0 & 7 & .1113 \\
\hline Vitreous hemorrhage & 8 & 0 & 8 & .0868 \\
\hline Recurrence & 56 & 11 & 44 & .1620 \\
\hline Type 2 choroidal neovascularization & 26 & 1 & 25 & .0030 \\
\hline Fibrosis & 31 & 4 & 27 & .0533 \\
\hline Serous retinal detachment & 81 & 20 & 65 & .0899 \\
\hline Subretinal hemorrhage (> 1DA) & 55 & 9 & 46 & .0157 \\
\hline Cystoid macula edema & 42 & 6 & 36 & .0266 \\
\hline Pigment epithelial detachment (> 1DA) & 41 & 5 & 36 & .0096 \\
\hline Tear of retinal pigment epithelium & 8 & 0 & 8 & .0868 \\
\hline \multicolumn{5}{|l|}{$\mathrm{n}=$ number; $\mathrm{DA}=$ disc area } \\
\hline \multicolumn{5}{|c|}{ One disc area (DA) is estimated as $2.54 \mathrm{~mm}^{2}$ on the basis of one optic disc diameter being $1.8 \mathrm{~mm}$. } \\
\hline \multicolumn{5}{|c|}{ Based on the area of the lesion at the initial visit, polypoidal choroidal vasculopathy (PCV) eyes were } \\
\hline
\end{tabular}


TABLE 3. ARMS2 Genotypes and Alleles in Patients with Polypoidal Choroidal Vasculopathy

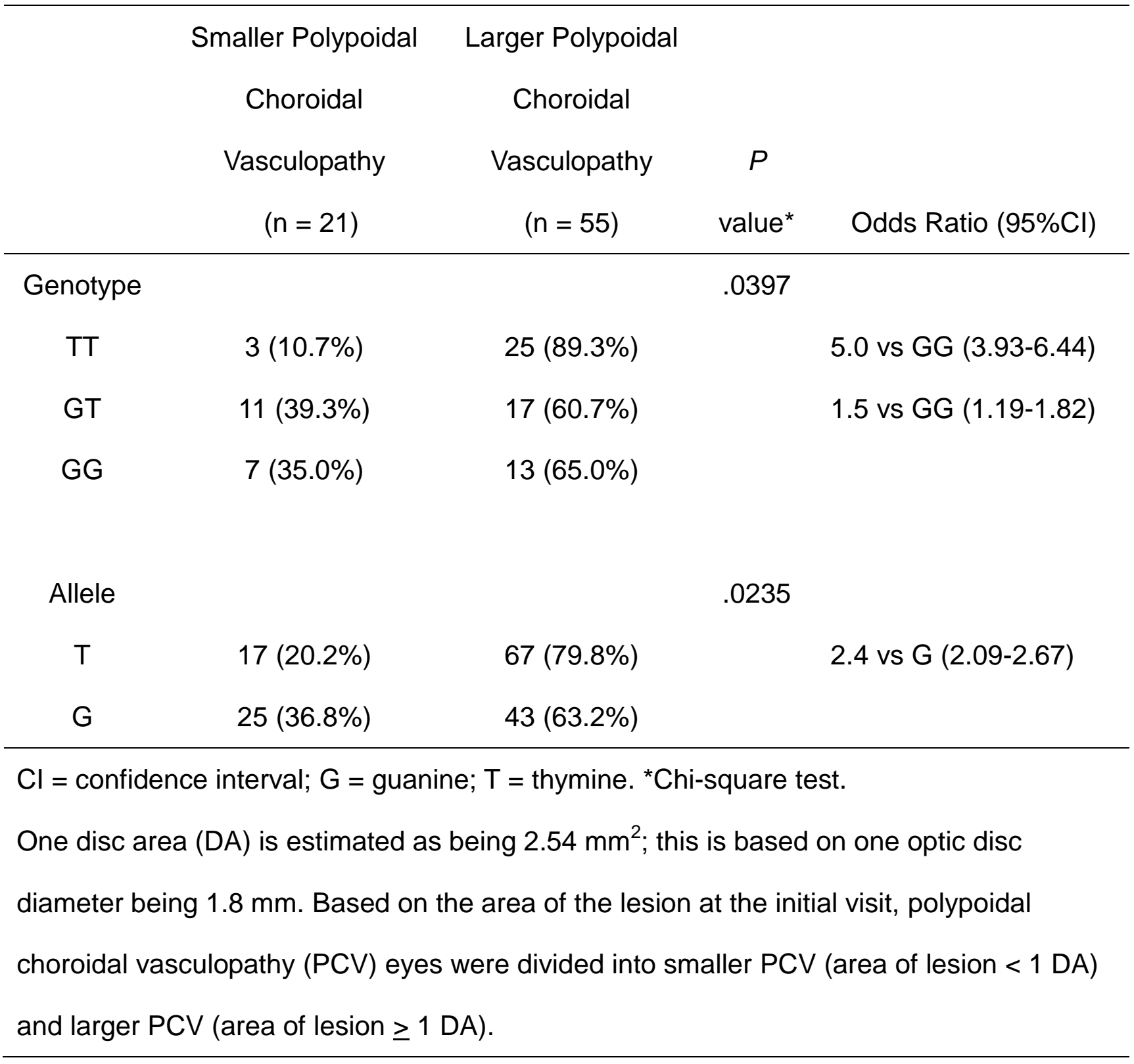



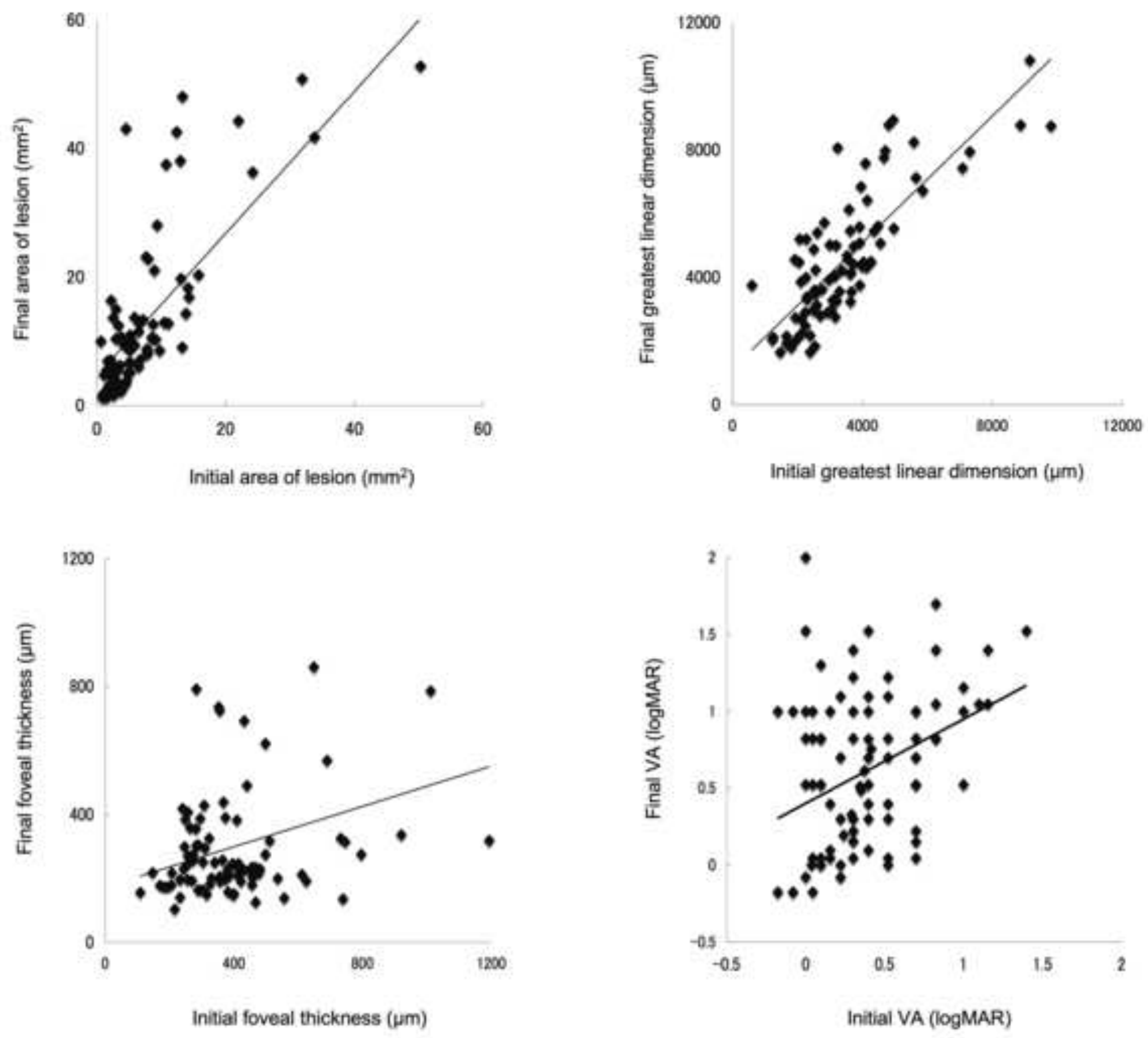

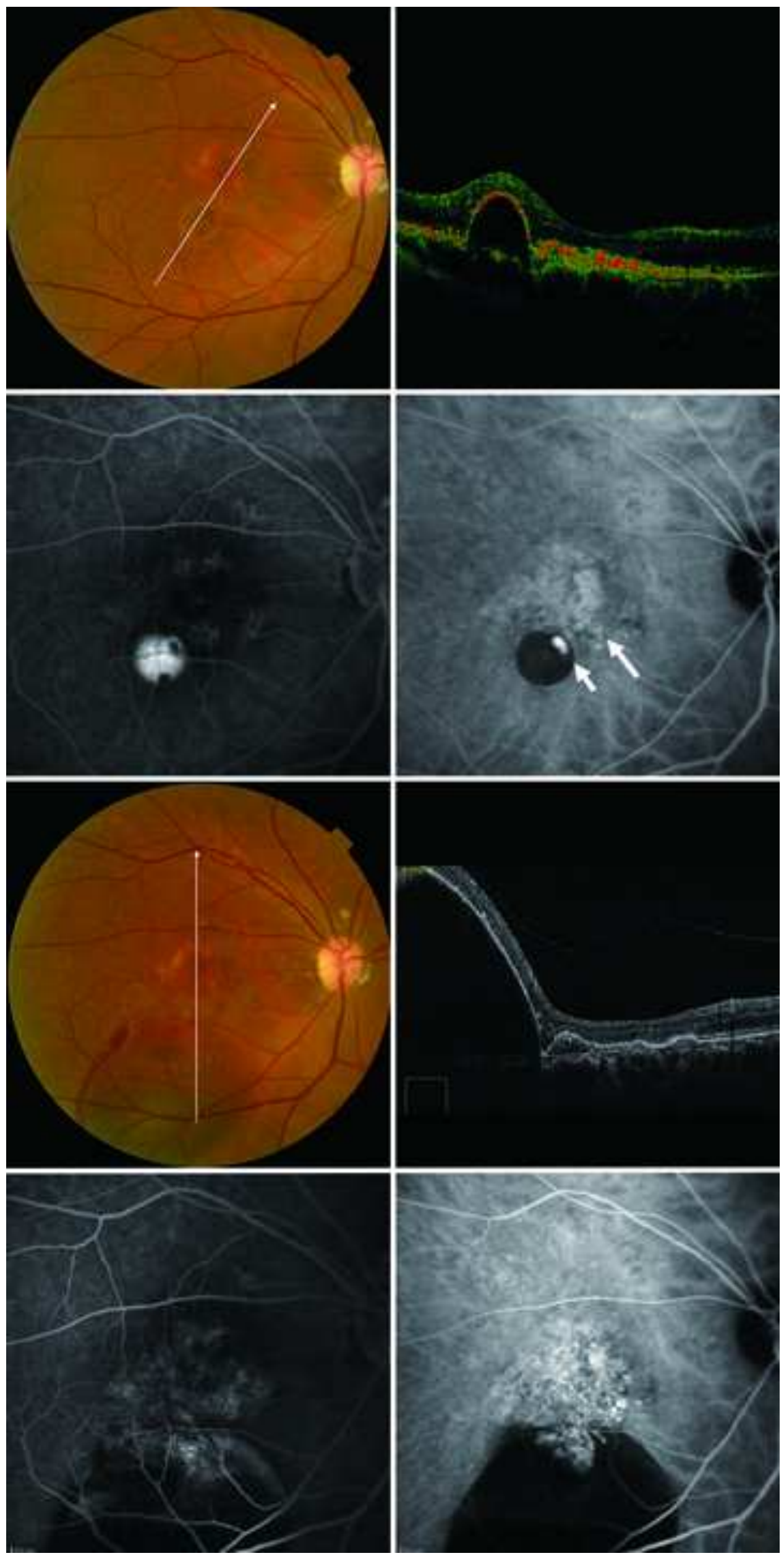

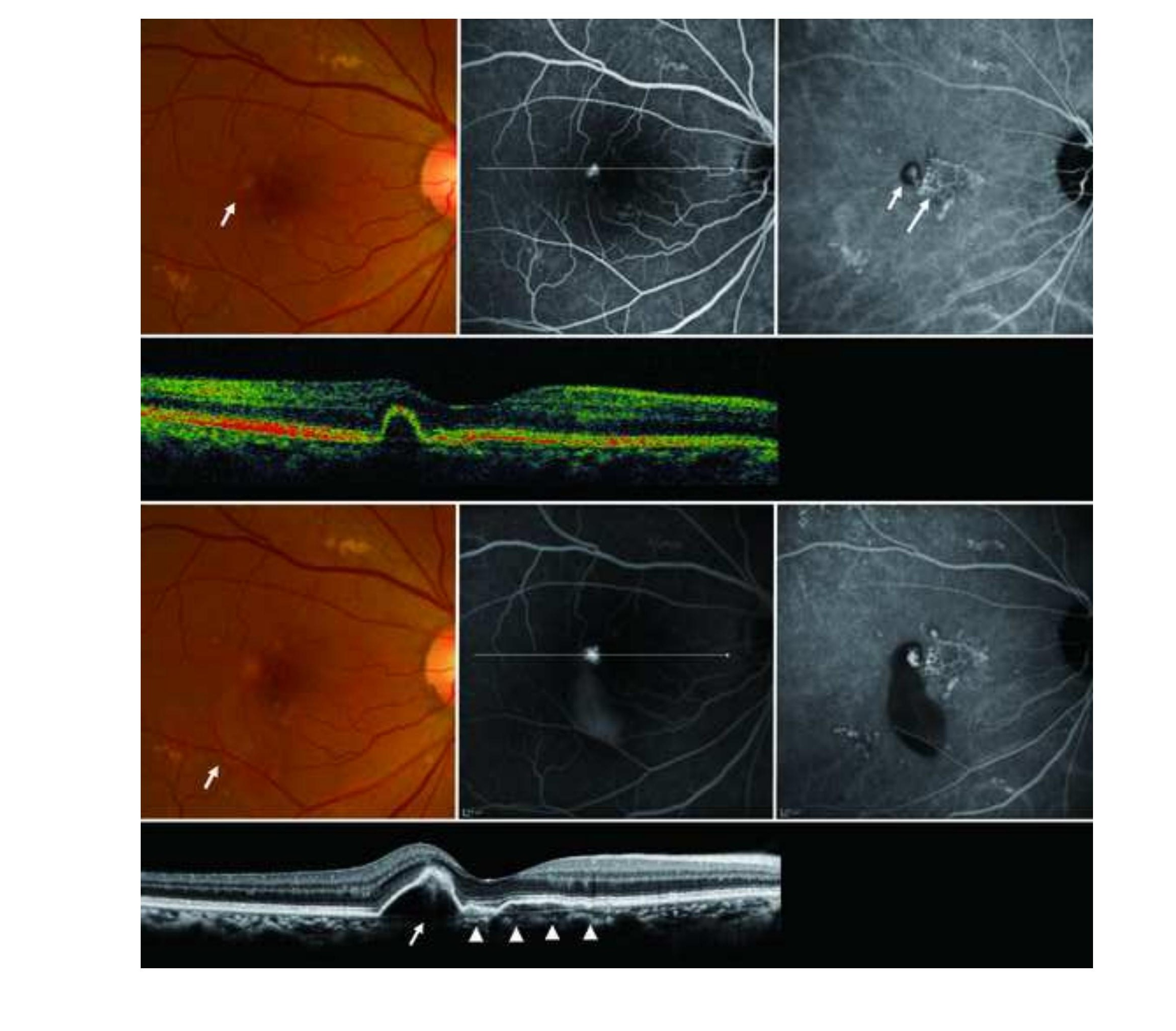

Figure 4.

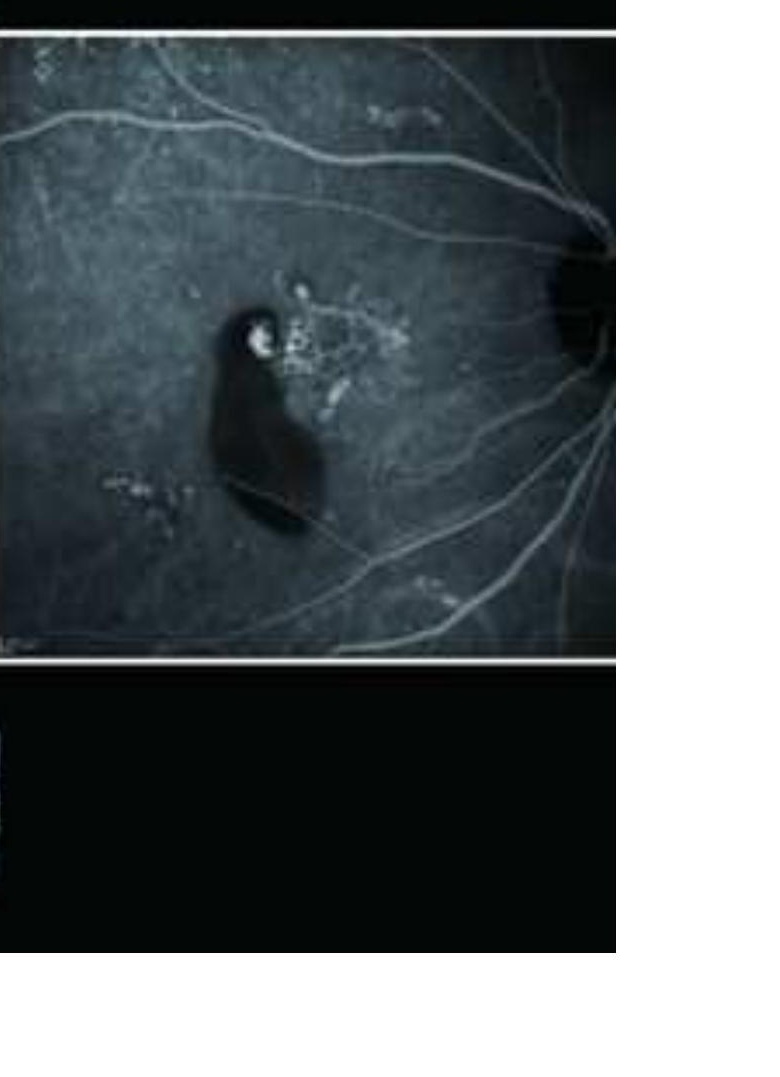

.
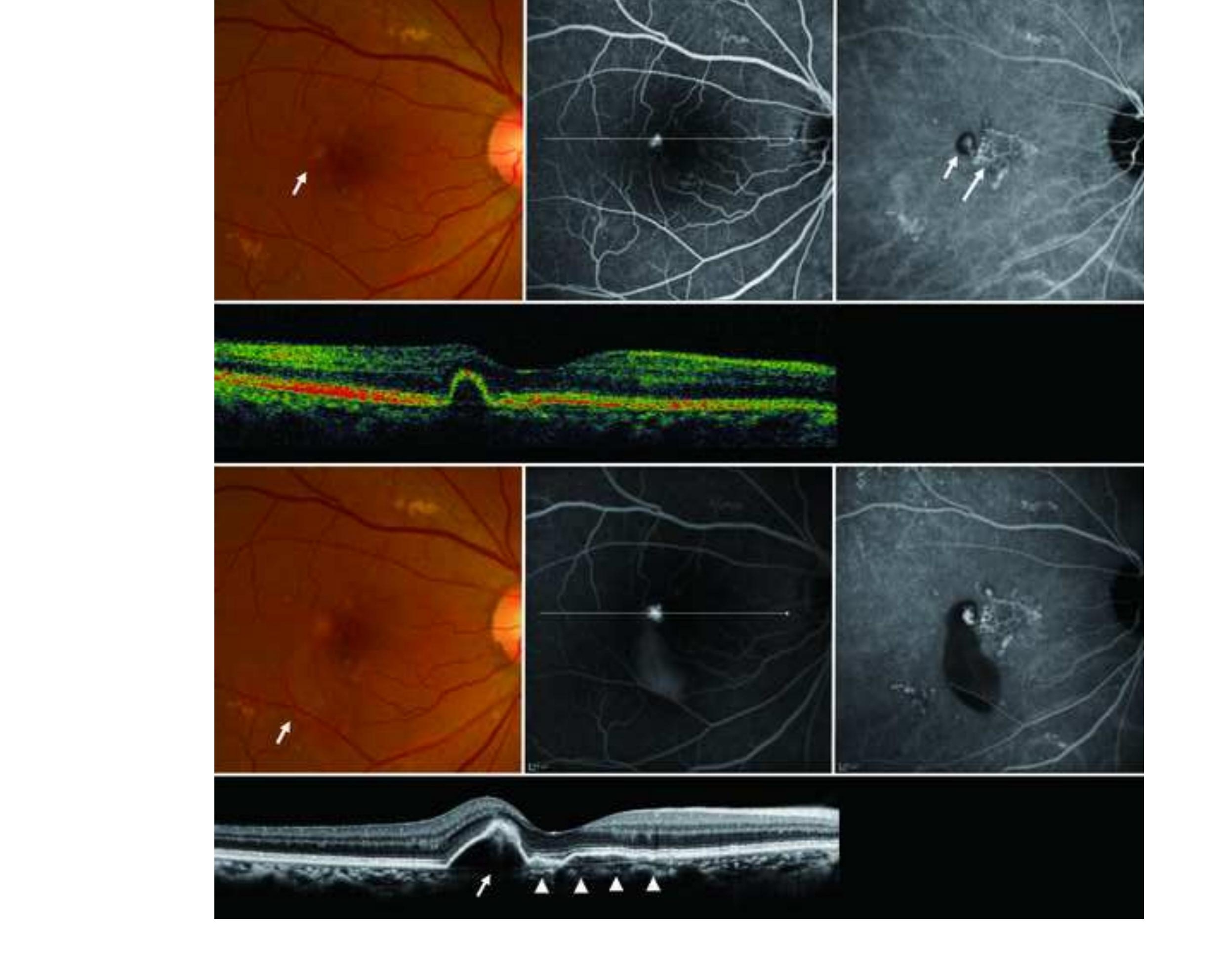

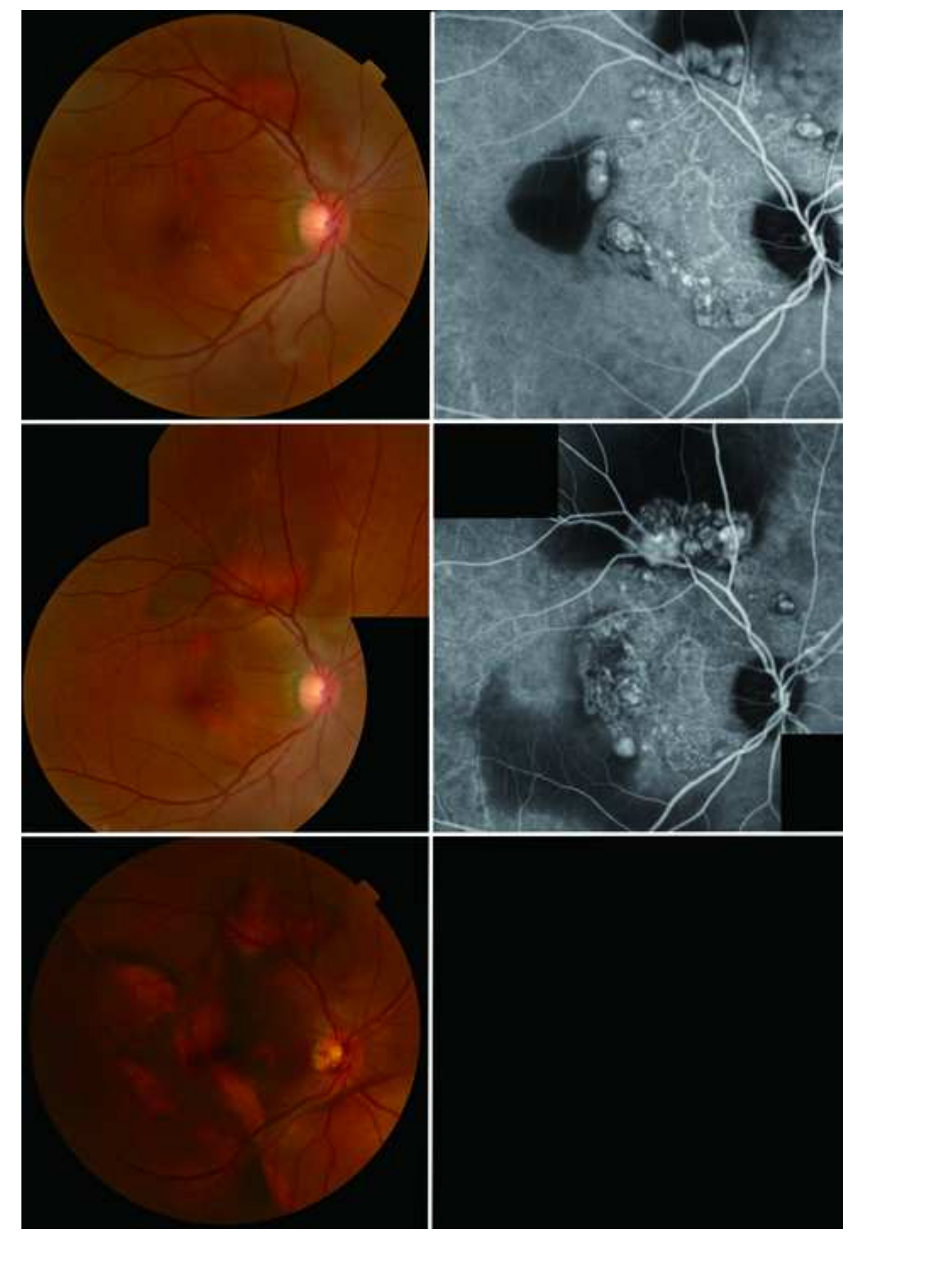

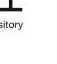

\section{KYOTOUNIVERSTY}

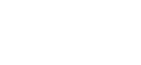

